\title{
QUOTIENTS OF BOUNDED OPERATORS
}

\author{
SAICHI IZUMINO \\ (Communicated by John B. Conway) \\ Dedicated to Professor Masahiro Nakamura on his 70th birthday
}

\begin{abstract}
We define a quotient $[B / A]$ of bounded operators $A$ and $B$ on a Hilbert space $H$ with $\operatorname{ker} A \subset \operatorname{ker} B$ as the mapping $A x \rightarrow B x, x \in H$, and show explicit formulae for computing quotients which correspond to sums, products, adjoints and closures of given quotients.
\end{abstract}

\section{INTRODUCTION}

Let $A$ and $B$ be bounded (linear) operators on a Hilbert space $H$ with the kernel condition

$\operatorname{ker} A \subset \operatorname{ker} B$.

We then define the quotient $[B / A]$ as the mapping $A x \rightarrow B x, x \in H$. If we write $G(A, B)$ for the set $\{(A x, B x) ; x \in H\}$ in the product Hilbert space $H \times H$, then $G(A, B)$ is a graph. We could define $[B / A]$ as the operator corresponding to this graph. A quotient (of bounded operators) so defined is, as a matter of fact, just what was called "opérateur $J$ uniforme" by Dixmier [2] and "semiclosed operator" by Kaufman [7], and several characterizations were given. It was proved in [7] that the class of quotients contains all closed operators in $H$ and is itself closed under sums and products.

In this paper we first show explicit formulae for computing quotients corresponding to the sum and the product of two given quotients, which really assures the algebraic closure property. We next present reasonable quotients which coincide with the adjoint and the closure of a given quotient if they exist, and deduce a fractional representation due to Kaufman [6] of a densely

Received by the editors August 2, 1988.

1980 Mathematics Subject Classification (1985 Revision). Primary 47A05; Secondary 47A99.

Key words and phrases. Closed operator, semiclosed operator, operator range, quotient of operators. 
defined closed operator. We also give some other applications which show the advantage of fractional representations of operators.

\section{Preliminaries}

First we state the majorization lemma due to Douglas [3] which is a key fact for our discussion.

Lemma 2.1 [4, Theorem 2.1]. Let $R$ and $S$ be bounded operators (on $H$ ). Then the following conditions are equivalent.

(1) $R H \subset S H$.

(2) $R R^{*} \leq \alpha S S^{*}$ for some $\alpha>0$.

(3) There exists a bounded operator $X$ such that $S X=R$.

With the restriction $\operatorname{ker} X^{*} \supset \operatorname{ker} S$, the solution $X$ of $S X=R$ in the above lemma is unique [4, p. 259]. We shall call this operator $X$ the Douglas solution, or shortly, D-solution.

It is useful to define the relation $[B / A] \subset[D / C]$ between two quotients $[B / A]$ and $[D / C]$, if $G(A, B) \subset G(C, D)$. In this case we say that $[D / C]$ is an extension of $[B / A]$, or $[B / A]$ is a restriction of $[D / C]$. Note that $G(A, B)$ and $G(C, D)$ are respectively the ranges of operators $\left(\begin{array}{ll}A & 0 \\ B & 0\end{array}\right)$ and $\left(\begin{array}{ll}C & 0 \\ D & 0\end{array}\right)$ on the Hilbert space $H^{\prime}:=H \times H$. Hence, applying Lemma 2.1 to those operators, we can obtain

Lemma 2.2. $[B / A] \subset[D / C]$ if and only if there exists a bounded operator $X$ such that $A=C X$ and $B=D X$.

To construct quotients corresponding to the sum and the product of two quotients, we need to show that for bounded operators $A$ and $B$ the sets of the form

$$
A H+B H, \quad A H \bigcap B H \quad \text { and } B^{-1}(A H):=\{x \in H ; B x \in A H\}
$$

are operator ranges, that is, ranges of some bounded operators. To this end we shall make use of a technique introduced by Fillmore and Williams [4]. Let $R$ (or more precisely $\left.R_{A, B}\right)=\left(A A^{*}+B B^{*}\right)^{1 / 2}$. Then as is well-known $R H=A H+B H$ [4, Theorem 2.2]. To obtain the remaining two kinds of operators we have to use the parallel sum of $A A^{*}$ and $B B^{*}$. Let $X$ and $Y$ be the D-solutions of $R X=A$ and $R Y=B$, respectively. Following [4] we put $A A^{*}: B B^{*}=A X^{*} Y B^{*}$ and call it the parallel sum of $A A^{*}$ and $B B^{*}$. Denote by $P$ the orthogonal projection onto the closure $(R H)^{-}$of $R H$. Then (3) and (4) in the following lemma present the desired operators.

Lemma 2.3 (cf. [4, Theorem 4.2]). Let $A$ and $B$ be bounded operators, and let $R, X, Y$ and $P$ be the operators as given above. Then

(1) $X X^{*}+Y Y^{*}=P$.

(2) $A A^{*}: B B^{*}=B B^{*}: A A^{*}=A\left(1-X^{*} X\right) A^{*}=B\left(1-Y^{*} Y\right) B^{*}$.

(3) $\left(A A^{*}: B B^{*}\right)^{1 / 2} H=A H \cap B H$.

(4) $\left(1-Y^{*} Y\right)^{1 / 2} H=B^{-1}(A H \cap B H)=B^{-1}(A H)$. 
Proof. (1) Put $Q=X X^{*}+Y Y^{*}$. Then by definition $R Q R=A A^{*}+B B^{*}=R^{2}$ or $R(Q-P) R=0$, that is, $(Q-P) R H \subset \operatorname{ker} R$. Since $\operatorname{ker} X^{*} \supset \operatorname{ker} R$, we have $X H \subset(\operatorname{ker} R)^{\perp}$, the orthogonal complement of $\operatorname{ker} R$. Similarly we have $Y H \subset(\operatorname{ker} R)^{\perp}$. Hence $(Q-P) R H \subset(Q-P) H \subset(\operatorname{ker} R)^{\perp}$, which implies that $(Q-P) R=0$ or $R(Q-P)=0$. Again by a similar argument as above we can obtain $Q-P=0$.

(2) Note that $A A^{*}: B B^{*}=R X X^{*} Y Y^{*} R$. Since $P X=X$ and $P Y=Y$, we can see from (1) that $X X^{*}$ and $Y Y^{*}$ commute. Now all equalities are easily obtained.

(3) Since $S H=\left(S S^{*}\right)^{1 / 2} H$ in general, it follows from (2) that

$$
\left(A A^{*}: B B^{*}\right)^{1 / 2} H=A\left(1-X^{*} X\right)^{1 / 2} H=B\left(1-Y^{*} Y\right)^{1 / 2} H .
$$

Hence we easily have $\left(A A^{*}: B B^{*}\right)^{1 / 2} H \subset A H \cap B H$. To see the converse inclusion, let $y \in A H \cap B H$. Then there exist $u, v \in H$ such that $y=A u=B v$. Hence $R(X u-Y v)=0$, so that $X^{*}(X u-Y v)=0$. Hence

$$
\begin{aligned}
u & =\left(1-X^{*} X\right) u+X^{*} Y v \in\left(1-X^{*} X\right) H+X^{*} Y H \\
& =\left\{\left(1-X^{*} X\right)^{2}+\left(X^{*} Y\right)\left(X^{*} Y\right)^{*}\right\}^{1 / 2} H=\left(1-X^{*} X\right)^{1 / 2} H .
\end{aligned}
$$

This implies that $y \in A\left(1-X^{*} X\right)^{1 / 2} H$.

(4) We can easily see that $B^{-1}(A H \cap B H)=B^{-1}(A H)$. For another equality, we first have, from (2.1) and (2), that

$$
\left(1-Y^{*} Y\right)^{1 / 2} H \subset B^{-1}\left(\left(A A^{*}: B B^{*}\right)^{1 / 2} H\right)=B^{-1}(A H \bigcap B H) .
$$

Next, if $u \in B^{-1}(A H \cap B H)$ then $B u \in B\left(1-Y^{*} Y\right)^{1 / 2} H$, or $u=\left(1-Y^{*} Y\right)^{1 / 2} v+$ $w$ for some $v \in H$ and $w \in \operatorname{ker} B$. Hence we now have only to show that $w \in\left(1-Y^{*} Y\right)^{1 / 2} H$. But we can see this from the fact that $\left(B^{*} H\right)^{-}=\left(Y^{*} H\right)^{-}$ or $\operatorname{ker} B=\operatorname{ker} Y$.

\section{SUMS AND PRODUCTS}

Let $[B / A]$ and $[D / C]$ be two quotients (with the respective kernel conditions of (1.1)). Then the sum of them is defined on the intersection of their domains $A H$ and $C H$. Hence we naturally choose $A * C:=\left(A A^{*}: C C^{*}\right)^{1 / 2}$ as the denominator of the sum. Now, for the quotient form of the sum we have

Theorem 3.1 (cf. [7, Theorem 3]). $[B / A]+[D / C]=\left[\left(B C_{1}+D A_{1}\right) / A * C\right]$.

Here $C_{1}$ and $A_{1}$ are, respectively, the D-solutions $X$ and $Y$ of $A X=A * C$ and $C Y=A * C$.

Proof. Note that $[B X / A * C]=[B X / A X]$ and $[D Y / A * C]=[D Y / C Y]$ are, respectively, restrictions of $[B / A]$ and $[D / C]$ to their common domain $(A *$ $C) H$. Hence the sum of the two quotients is the mapping $(A * C) u \rightarrow B X u+$ $D Y u, u \in H$. 
Remark. In the above theorem, clearly we can adopt any operator $E$ with $E H=A H \cap C H$ instead of $A * C$. To construct the corresponding numerator of the sum, we can choose arbitrary solutions $X$ and $Y$, respectively, instead of the D-solutions, because $B X$ and $D Y$ are well-defined by the respective kernel conditions for the two quotients.

The product $[B / A][D / C]$ is defined as the operator $C u \rightarrow B v$ for $u, v \in H$ such that $D u=A v$. Hence the domain of the product is $\left\{C u ; u \in D^{-1}(A H)\right\}=$ $C D^{-1}(A H)$. Let $R_{A, D}=\left(A A^{*}+D D^{*}\right)^{1 / 2}$ and let $Y$ be the D-solution of the equation $R_{A, D} Y=D$. Then from Lemma 2.3(4) (replacing $B$ by $D$ ), we have $D^{-1}(A H)=\left(1-Y^{*} Y\right)^{1 / 2} H$, so that $C D^{-1}(A H)=C A_{2} H$ if we put $A_{2}=\left(1-Y^{*} Y\right)^{1 / 2}$. Now, for the product of two quotients we have

Theorem 3.2 (cf. [7, Theorem 3]). $[B / A][D / C]=\left[B D_{2} / C A_{2}\right]$.

Here $A_{2}$ is the operator as given above and $D_{2}$ is the D-solution $Z$ of $A Z=D A_{2}$.

Proof. Since the domain of the product $[B / A][D / C]$ is $C A_{2} H$, we see that the composition

$$
C A_{2} u \rightarrow D A_{2} u=A Z u \rightarrow B Z u, \quad u \in H,
$$

determines the desired product.

Remark. In Theorem 3.2 we can replace $A_{2}$ by any operator $F$ satisfying $F H=D^{-1}(A H)$. We can also choose any solution $Z$ instead of the D-solution for the same reason as in the case of the sum.

\section{ADJOINTS AND CLOSURES}

Let $[B / A]$ be a quotient densely defined, that is, a quotient with the domain $A H$ dense in $H$, and let $G(A, B)$ be its graph. Then the adjoint $G(A, B)^{*}$ is naturally defined as the set of elements $(x, y)$ in $H \times H$ such that $\langle B u, x\rangle=$ $\langle A u, y\rangle$ for all $u \in H .(\langle\cdot, \cdot\rangle$ is the inner product in $H$.$) We can see that$ $G(A, B)^{*}=\left\{(x, y) ; B^{*} x=A^{*} y\right\}$ and it is a graph again. The corresponding operator is just the adjoint of $[B / A]$, that is, $[B / A]^{*}$. The domain of $[B / A]^{*}$ is hence $B^{*(-1)}\left(A^{*} H\right)$, so that by Lemma 2.3(4) the set is the range of the operator $A_{*}:=\left(1-Y^{*} Y\right)^{1 / 2}$, where $Y$ is the D-solution of the equation $R_{A^{*}, B^{*}} Y=B^{*}$ $\left(R_{A^{*}, B^{*}}=\left(A^{*} A+B^{*} B\right)^{1 / 2}\right)$. For the convenience sake we write $R_{l}=R_{A^{*}, B^{*}}$ and denote by $B_{l}$ the (unique) solution $Z$ of $Z R_{l}=B, \operatorname{ker} Z \supset \operatorname{ker} R_{l}$. Then it is clear that $Z=Y^{*}=B_{l}$, and hence $A_{*}=\left(1-Z Z^{*}\right)^{1 / 2}=\left(1-B_{l} B_{l}^{*}\right)^{1 / 2}$. (Similarly to $B_{l}$ we denote by $A_{l}$ the solution $W$ of $W R_{l}=A, \operatorname{ker} W \supset$ $\operatorname{ker} R_{l}$.) Note that $\operatorname{ker} A^{*}=\{0\}$. Hence we can obtain a bounded operator $X$ uniquely determined by $B^{*} A_{*}=A^{*} X$, and then the mapping $x=A_{*} u \rightarrow$ $X u=y, u \in H$ is just the adjoint $[B / A]^{*}$. Unlike $A_{*}$ we define $B_{*}$ as 
the operator $X$ (not $\left(1-A_{l} A_{l}^{*}\right)^{1 / 2}$ ) for formal construction of the quotient representing $[B / A]^{*}$. Now we have

Theorem 4.1. Let $[B / A]$ be a densely defined quotient. Then $[B / A]^{*}=\left[B_{*} / A_{*}\right]$. Here $A_{*}=\left(1-B_{l} B_{l}^{*}\right)^{1 / 2}$ and $B_{*}$ is the unique solution $X$ of $A^{*} X=B^{*} A_{*}$. Explicitly, $B_{*}=V_{l} B_{l}^{*}$, where $V_{l}$ is the partial isometry obtained from the polar decomposition $A_{l}=V_{l}\left(A_{l}^{*} A_{l}\right)^{1 / 2}$ of $A_{l}$.

Proof. It suffices to show that $B_{*}=V_{l} B_{l}^{*}$. Denote by $P_{l}$ the orthogonal projection onto $\left(R_{l} H\right)^{-}$. Then by Lemma $2.3(1)$ we can see

$$
A_{l}^{*} A_{l}+B_{l}^{*} B_{l}=P_{l} .
$$

By definition $A_{l} R_{l}=A$ and $B_{l} R_{l}=B$. Furthermore, we easily have $\left(A_{l}^{*} A_{l}\right)^{1 / 2}$ $=A_{l}^{*} V_{l}$. Hence now

$$
\begin{aligned}
A^{*} B_{*} & =B^{*} A_{*}=R_{l} B_{l}^{*}\left(1-B_{l} B_{l}^{*}\right)^{1 / 2}=R_{l}\left(P_{l}-B_{l}^{*} B_{l}\right)^{1 / 2} B_{l}^{*} \\
& =R_{l}\left(A_{l}^{*} A_{l}\right)^{1 / 2} B_{l}^{*}=R_{l} A_{l}^{*} V_{l} B_{l}^{*}=A^{*} V_{l} B_{l}^{*} .
\end{aligned}
$$

Hence $B_{*}=V_{l} B_{l}^{*}$, because $\operatorname{ker} A^{*}=\{0\}$.

Remark. Let $C$ and $D$ be bounded operators such that $C H=A_{*} H$ and $B^{*} C=A^{*} D$. Then by the definition of the adjoint we can see $[B / A]^{*}=[D / C]$.

To construct the second adjoint $[B / A]^{* *}=\left[B_{*} / A_{*}\right]^{*}$ we have to assume that $A_{*} H$ is dense in $H$. Since $[B / A]^{* *}$ is then the closure of $[B / A]$, this assumption is nothing but the condition for $[B / A]$ to be closable. First, related to this condition we have

Theorem 4.2. Let $[B / A]$ be a densely defined quotient. Then the following conditions are equivalent.

(1) $[B / A]$ is closable. $\left(A_{*} H\right.$ is dense in $H$.)

(2) $\operatorname{ker} A_{l} \subset \operatorname{ker} B_{l}$.

(3) $A_{*}^{2}+B_{*}^{*} B_{*}=1$.

Proof. (1) $\Rightarrow$ (2). Let $A_{l} u=0$. Then from (4.1) we have $A_{*}^{2} B_{l} u=$ $\left(1-B_{l} B_{l}^{*}\right) B_{l} u=B_{l}\left(P_{l}-B_{l}^{*} B_{l}\right) u=B_{l} A_{l}^{*} A_{l} u=0$. Hence $B_{l} u \in \operatorname{ker} A_{*}=\{0\}$.

(2) $\Rightarrow(3)$. Since $\operatorname{ker} A_{l}=\left(1-V_{l}^{*} V_{l}\right) H$, we have $B_{l}\left(1-V_{l}^{*} V_{l}\right) H=\{0\}$, or $B_{l}=B_{l} V_{l}^{*} V_{l}$. Hence $A_{*}^{2}+B_{*}^{*} B_{*}=1-B_{l} B_{l}^{*}+B_{l} V_{l}^{*} V_{l} B_{l}^{*}=1$.

(3) $\Rightarrow(1)$. Let $A_{*} u=0$. Then we have to show $u=0$. Since $\operatorname{ker} A_{*} \subset \operatorname{ker} B_{*}$, we have $B_{*} u=0$, so that $u=\left(A_{*}^{2}+B_{*}^{*} B_{*}\right) u=0$.

Remark. Every quotient is not closable; so let $P_{*}$ be the orthogonal projection onto $\left(A_{*} H\right)^{-}$. Then with the same assumption as in Theorem 4.2 we have

$$
A_{*}^{2}+B_{*}^{*} B_{*}=P_{*} \text {. }
$$

Proof. From the identity $B_{*}=V_{l} B_{l}^{*}$ and (4.1) we can see $B_{*} A_{*}=A_{l} B_{l}^{*}$. Using this fact and (4.1) again, we can show that $A_{*}\left(A_{*}^{2}+B_{*}^{*} B_{*}\right) A_{*}=A_{*}^{2}$, or 
$A_{*} Q A_{*}=0$, where $Q=A_{*}^{2}+B_{*}^{*} B_{*}-P_{*}$. On the other hand we easily see that $Q H \subset\left(\operatorname{ker} A_{*}\right)^{\perp}$. Hence we can obtain $Q=0$, say, by a similar argument as in the proof of Lemma 2.3(1).

Now, on the second adjoint or closure of a quotient we have

Theorem 4.3. Let $[B / A]$ be a densely defined, closable quotient. Then $[B / A]^{* *}=$ $\left[B_{*}^{*} /\left(1-B_{*} B_{*}^{*}\right)^{1 / 2}\right]=\left[B_{l} / A_{l}\right]$.

Proof. By definition $[B / A]^{* *}=\left[B_{*} / A_{*}\right]^{*}=\left[B_{* *} / A_{* *}\right]$. By Theorem 4.2(3) we can see that $A_{* l}\left(=\left(A_{*}\right)_{l}\right)=A_{*}$ and $B_{* l}=B_{*}$, so that $A_{* *}=\left(1-B_{*} B_{*}^{*}\right)^{1 / 2}$. Further, let $X$ be the unique solution of $A_{*} X=B_{*}^{*} A_{* *}$. Then, since $A_{*} X=$ $B_{*}^{*}\left(1-B_{*} B_{*}^{*}\right)^{1 / 2}=\left(1-B_{*}^{*} B_{*}\right)^{1 / 2} B_{*}^{*}=A_{*} B_{*}^{*}$, we have $X=B_{*}^{*}$, or $B_{* *}=B_{*}^{*}$ by definition. Hence we have the first equality. For the other equality we first note that $\left[B_{l} / A_{l}\right]$ is a quotient by Theorem 4.2(2). Next since $A_{l} H$ is dense in $H$, we have $V_{l} V_{l}^{*}=1$. From this identity and (4.1) we can see that $A_{l} H=\left(V_{l} A_{l}^{*} A_{l} V_{l}^{*}\right)^{1 / 2} H=\left(1-B_{*} B_{*}^{*}\right)^{1 / 2} H$ and $A_{*} B_{l}=B_{*}^{*} A_{l}$. Hence by the remark after Theorem 4.1 we have $\left[B_{*} / A_{*}\right]^{*}=\left[B_{l} / A_{l}\right]$.

Remarks. (1) If $[B / A]$ is closable, then $G\left(A_{l}, B_{l}\right)$ is a graph by Theorem 4.2(2). Hence we could show $[B / A]^{* *}=\left[B_{l} / A_{l}\right]$ from the fact $G\left(A_{l}, B_{l}\right)=G(A, B)^{-}$. (2) If $[B / A]$ is closed, then $R_{l} H$ is closed in $H$ [6, Theorem 1]. In this case we have $A_{l}=A X$ and $B_{l}=B X$ for a generalised inverse $X$ of $R_{l}$ (say, [1, p. 321]). Hence we can easily see that $\operatorname{ker} A_{l} \subset \operatorname{ker} B_{l}$ and $[B / A]=\left[B_{l} / A_{l}\right]$ (say, by Lemma 2.2).

When an operator $T$ is densely defined and closed, then $T$ is represented as a quotient (say, [11, p. 307]). Since $T=T^{* *}$, we can write $T=$ $\left[B_{*}^{*} /\left(1-B_{*} B_{*}^{*}\right)^{1 / 2}\right]$ with some operator $B_{*}$ by Theorem 4.3. Hence, putting $B_{*}^{*}=C$, we now obtain the following fractional representation of a closed operator due to Kaufman. (His notation is $C\left(1-C^{*} C\right)^{-1 / 2}$.)

Corollary 4.4 [6, Corollary]. Let $T$ be a densely defined closed operator. Then there exists a pure contraction $C$, that is, contraction with $\operatorname{ker}\left(1-C^{*} C\right)=\{0\}$, such that $T=\left[C /\left(1-C^{*} C\right)^{1 / 2}\right]$. Furthermore, the operator $C$ is uniquely determined by $T$.

Proof. It suffices to show uniqueness of $C$. Let $\left[C /\left(1-C^{*} C\right)^{1 / 2}\right]=$ $\left[D /\left(1-D^{*} D\right)^{1 / 2}\right]$ for two pure contractions $C$ and $D$. Then $\left(1-C^{*} C\right)^{1 / 2}=$ $\left(1-D^{*} D\right)^{1 / 2} X$ and $C=D X$ for some invertible operator $X$ by Lemma 2.2 . We can easily obtain $X^{*} X=1$. Hence $X$ is unitary, so that $1-C^{*} C=$ $\left(1-D^{*} D\right)^{1 / 2} X X^{*}\left(1-D^{*} D\right)^{1 / 2}=1-D^{*} D$, or $C^{*} C=D^{*} D$. Hence $X=1$, and $C=D$.

With the same notation as before we can show the following fact, say, from Theorem 4.1 and the identity $B_{*}^{*} B_{*}=B_{l} B_{l}^{*}$. 
Corollary 4.5 $\left[8\right.$, Lemma 1]. $T^{*}=\left[C^{*} /\left(1-C C^{*}\right)^{1 / 2}\right]$.

\section{SOME APPLiCATIONS}

In this section we apply quotients to obtain some new results and new proofs of known results.

I. Bounded quotients. A quotient $[B / A]$ is a restriction to $A H$ of a bounded operator when $B^{*} H \subset A^{*} H$. In fact, if $B^{*}=A^{*} X$ or $B=X^{*} A$ for a bounded operator $X$, then $[B / A]=\left[X^{*} A / A\right] \subset\left[X^{*} / 1\right]=X^{*}$. We call such a quotient as $[B / A]$ bounded. Motivated by Okazaki [9] and Ôta [10], we show two theorems on bounded quotients.

Theorem 5.1 (cf. [9, Theorem 3]). Let $[B / A]$ be a quotient with $B H \subset A H$. If there exists $\lambda>0$ such that $(\mu A-B) H$ is closed in $H$ for $\mu>\lambda$, then $[B / A]$ is bounded.

Proof. There exists a bounded operator $C$ such that $A C=B$. Hence, if $\mu>\|C\|+\lambda$, then $\mu-C$ is invertible, and $A H=A(\mu-C) \cdot(\mu-C)^{-1} H=$ $(\mu A-B) H$. Hence $A H$ is closed, so that $A^{*} H$ is closed. Hence $B^{*} H \subset$ $(\operatorname{ker} B)^{\perp} \subset(\operatorname{ker} A)^{\perp}=A^{*} H$, which implies that $[B / A]$ is bounded.

Theorem 5.2 (cf. [10, Theorem 3.3]). Let $[B / A]$ be a densely defined closed quotient, and let $[B / A]^{*}=\left[B_{*} / A_{*}\right]$ be its adjoint. If $B_{*} H \subset A H$, then $[B / A]$ is bounded, so that it extends to a bounded operator on $H$.

Proof. Since $[B / A]$ is closed, we see that $[B / A]=\left[B_{*}^{*} /\left(1-B_{*} B_{*}^{*}\right)^{1 / 2}\right]$ by Theorem 4.3. Hence, by the assumption we have $B_{*} H \subset A H=\left(1-B_{*} B_{*}^{*}\right)^{1 / 2} H$, so that $B_{*} B_{*}^{*} \leq \alpha\left(1-B_{*} B_{*}^{*}\right)$ for some $\alpha>0$ by Lemma 2.1 . Hence $B_{*} B_{*}^{*} \leq$ $\alpha /(1+\alpha)<1$, and $1-B_{*} B_{*}^{*}$ is invertible. Hence $[B / A]$ is bounded.

II. Normal operators. As one of the preservative properties of the function $\Gamma(C):=C\left(1-C^{*} C\right)^{-1 / 2}$ ( $C$ is a pure contraction), Kaufman [8] proved that $\Gamma(C)$ is normal if and only if $C$ is normal. Using the product formula of quotients, we give an alternative proof to this fact (and a result in Stone [12]).

Theorem 5.3 (cf. [8, Theorem 2], [12, Theorem 6]). Let $T=\left[C /\left(1-C^{*} C\right)^{1 / 2}\right]$ be a densely defined closed operator. Then the following conditions are equivalent.

(1) $T$ is normal, that is, $T^{*} T=T T^{*}$.

(2) $T^{*} T \subset T T^{*}$

(3) $T^{*} T \supset T T^{*}$.

(4) $C$ is normal.

Proof. By the formula of the product we can see that $T^{*} T=\left[C^{*} /\left(1-C C^{*}\right)^{1 / 2}\right] \times$ $\left[C /\left(1-C^{*} C\right)^{1 / 2}\right]=\left[C^{*} C /\left(1-C^{*} C\right)\right]$. Similarly, we can also see that $T T^{*}=$ $\left[C C^{*} /\left(1-C C^{*}\right)\right]$. Hence, if $T^{*} T \subseteq T T^{*}$, then we have $1-C^{*} C=\left(1-C C^{*}\right) X$ and $C^{*} C=C C^{*} X$ for some operator $X$. We now immediately have $X=1$. Hence $C$ is normal. Conversely, if $C$ is normal, then clearly $T^{*} T=T T^{*}$. In case $T^{*} T \supset T T^{*}$, we can still obtain the same conclusion as before. 
III. Decomposition into the closable part and the singular part. In [5] Jorgensen introduced the decomposition of a (densely defined) operator $T$ into the sum of its closable part $T_{c}:=Q T$ and its singular part $T_{s}:=Q^{\perp} T\left(Q^{\perp}=1-Q\right)$, where $Q$ is the orthogonal projection onto the closure of the domain of $T^{*}$ [5, p. 285]. If $T=[B / A]$, a densely defined quotient, then we have $Q=$ $P_{*}=\left[P_{*} / 1\right], T_{c}=\left[P_{*} / 1\right][B / A]=\left[P_{*} B / A\right]$ and $T_{s}=\left[P_{*}^{\perp} / 1\right][B / A]=\left[P_{*}^{\perp} B / A\right]$. Furthermore, using (4.1) and (4.2) we can obtain that $P_{*} B=B_{l} V_{l}^{*} V_{l} R_{l}$. Hence we have

Theorem 5.4. Let $[B / A]$ be a densely defined quotient. Then

$$
\begin{aligned}
{[B / A] } & =\left[P_{*} B / A\right]+\left[P_{*}^{\perp} B / A\right] \\
& =\left[B_{l} V_{l}^{*} V_{l} R_{l} / A\right]+\left[\left(B-B_{l} V_{l}^{*} V_{l} R_{l}\right) / A\right]
\end{aligned}
$$

is its sum decomposition into the closable part and the singular part in the sense of Jorgensen.

Related to the above decomposition we have

$$
P_{*}^{\perp} B=0
$$

as an equivalent condition for $[B / A]$ to be closable. Since $\operatorname{ker} B^{*} \subset B^{*(-1)}$ $\left(A^{*} H\right)=A_{*} H \subset P_{*} H$, we can see that (5.1) (or $B^{*} P_{*}^{\perp}=0$ ) is really equivalent to the identity $P_{*}^{\perp}=0$, or $P_{*}=1$, the condition (1) Theorem 4.2.

As an extremely nonclosable quotient we call $[B / A]$ singular if $P_{*}^{\perp} B=B$. Now we show a characterization of such a quotient.

Theorem 5.5. Let $[B / A]$ be a densely defined quotient. Then the following conditions are equivalent.

(1) $[B / A]$ is singular.

(2) $A_{l} B_{l}^{*}=0$.

(3) $A^{*} H \cap B^{*} H=\{0\}$.

Proof. (1) $\Rightarrow(2)$. Since (1) is equivalent to $B H \subset \operatorname{ker} A_{*}$, we see that $A_{*} B=$ 0 , or $B^{*} A_{*}=0$. Hence $R_{l} A_{l}^{*} A_{l} B_{l}^{*}=R_{l}\left(P_{l}-B_{l}^{*} B_{l}\right) B_{l}^{*}=R_{l} B_{l}^{*}\left(1-B_{l} B_{l}^{*}\right)=$ $B^{*} A_{*}^{2}=0$. Hence, using $\operatorname{ker} R_{l} \subset \operatorname{ker} B_{l}$, we have $\left(A_{l} B_{l}^{*}\right)^{*}\left(A_{l} B_{l}^{*}\right)=B_{l} A_{l}^{*} A_{l} B_{l}^{*}$ $=0$, so that $A_{l} B_{l}^{*}=0$.

(2) $\Rightarrow(3)$. From Lemma 2.3(3) we see that (3) is equivalent to

$$
A^{*} A: B^{*} B=0 \text {. }
$$

Hence by the definition $A^{*} A: B^{*} B=A^{*} A_{l} B_{l}^{*} B$, the relation (2) $\Rightarrow(3)$ is clear. (3) $\Rightarrow(1)$. Note that $\left(A_{*} B\right)^{*}\left(A_{*} B\right)=B^{*} A_{*}^{2} B=B^{*}\left(1-B_{l} B_{l}^{*}\right) B=A^{*} A: B^{*} B$. Hence, from $\left(3^{\prime}\right)$ we have $A_{*} B=0$, that is, $B H \subset \operatorname{ker} A_{*}$. 


\section{REFERENCES}

1. A. Ben-Israel and T. N. Greville, Generalized inverses: theory and applications, Wiley, New York, 1974.

2. J. Dixmier, Étude sur les variétés et les opérateurs de Julia, Bull. Soc. Math. France 77 (1949), 11-101.

3. R. G. Douglas, On majorization, factorization and range inclusion of operators in Hilbert space, Proc. Amer. Math. Soc. 17 (1966), 413-416.

4. P. A. Fillmore and J. P. Williams, On operator ranges, Advances in Math. 7 (1971), 254-281.

5. P. E. T. Jorgensen, Unbounded operators: perturbations and commutativity problems, J. Functional Anal. 39 (1980), 281-307.

6. W. E. Kaufman, Representing a closed operator as a quotient of continuous operators, Proc. Amer. Math. Soc. 72 (1978), 531-534.

7. __ Semiclosed operators in Hilbert space, Proc. Amer. Math. Soc. 76 (1979), 67-73.

8. Closed operators and pure contractions in Hilbert space, Proc. Amer. Math. Soc. 87 (1983), 83-87.

9. Y. Okazaki, Boundedness of closed linear operator $T$ satisfying $R(T) \subset D(T)$, Proc. Japan Acad. 62 Ser. A (1986), 294-296.

10. S. Ôta, Closed linear operators with domain containing their range, Proc. Edinburgh Math. Soc. 27 (1984), 229-233.

11. F. Riesz and B. Sz.-Nagy, Functional analysis, Ungar, New York, 1955.

12. M. H. Stone, On unbounded operators in Hilbert space, J. Indiana Math. Soc. 15 (1951), 155-192.

Faculty of Education, Toyama University, 3190 Gofuku, Toyama-shi 930, Japan 